\title{
Birefringence of cellotape: Jones representation and experimental analysis
}

\author{
Augusto Beléndez $^{1,3}$, Elena Fernández ${ }^{2,3}$, Jorge Francés ${ }^{1}$ and CristianNeipp ${ }^{1,3}$ \\ ${ }^{1}$ Departamento de Física, Ingeniería de Sistemas y Teoría de la Señal. \\ Universidad de Alicante. Apartado 99. E-03080 Alicante. SPAIN \\ ${ }^{2}$ Departamento de Óptica, Farmacología y Anatomía. \\ Universidad de Alicante. Apartado 99. E-03080 Alicante. SPAIN \\ ${ }^{3}$ Instituto Universitario de Física Aplicada a las Ciencias y las Tecnologías. \\ Universidad de Alicante. Apartado 99. E-03080 Alicante. SPAIN
}

\begin{abstract}
In this paper we analyze a simple experiment to study the effects of polarized light. A simple optical system composed of a polarizer, a retarder (cellotape) and an analyzer is used to study the effect on the polarization state of the light which impinges on the setup. The optical system is characterized by means of a Jones matrix, and a simple procedure based on Jones vectors is used to obtain an expression for the intensity after the light passes through the optical system. The light intensity is measured by a photodetector and the expression obtained theoretically is experimentally validated. By fitting the experimental intensity data the value of the retardation introduced by the retarder can also be obtained.
\end{abstract}

KEYWORDS: Birefringence, retarder, Jones representation. 
A. Beléndez, E. Fernández, J. Francés and C. Neipp, "Birefringence of cellotape: Jones representation and experimental analysis", European Journal of Physics, Vol. 31, № 3, pp. 551-561 (2010). doi:10.1088/0143-0807/31/3/012

ISSN 0143-0807 (Print) ISSN 1361-6404 (Online)

\section{Introduction}

Light is a transversal electromagnetic wave and can therefore be polarized in different ways. Students are usually familiarized with linear polarization, the use of linear polarizers and Malus's law. Nevertheless, it is not easy for them to understand concepts such as circular or elliptic polarized light, birefringence or the action of retarders. Birefringence was discovered in 1669 by Erasmus Bartholinus (1625-1698) using calcite, which is called Iceland spar in older textbooks [1,2]. This phenomenon was first explained in 1690 by Huygens, who used the concept of an elliptical secondary wave [1, 2]. As described in Guenther's book [2], Bartholinus believed that the behaviour of calcite was a special case of refraction and thus called the effect 'double refraction'. It is important to remember that 'refringence' is an archaic synonym for 'refraction' and so its derivative, 'birefringence', is the term commonly used for 'double refraction' [2]. Retarders are devices which modify the polarization of an incident wave changing the relative phase of two orthogonally polarized waves that form the incident wave, and birefringence can be used to produce the desired phase change [2]. To understand how birefringent retarders work, we consider a uniaxial, birefringent plate cut with the optic axis parallel to the plane of the plate. When a beam of linearly polarized light passes normally through this plate, the wave can be decomposed into two components, one of them linearly polarized parallel to the optic axis of the plate and the other polarized perpendicular to this axis $[2,3]$. These waves are the ordinary and extraordinary waves in the birefringent plate and they propagate parallelly inside the crystal at different velocities. Hence, one wave is retarded relative to the other. If the retarder plate has a thickness $d$, these two waves emerge from the crystal with a phase difference given by the following expression [2]

$$
\Gamma=\frac{2 \pi}{\lambda_{0}} \Delta n d
$$

where $\lambda_{0}$ is the wavelength of the incident light in air and $\Delta n$ is the difference in the refractive indices of the crystal for these two components [3]. A retarder can be used to 
rotate the direction of linear polarization or convert linearly polarized light into elliptically polarized light $[2,4]$.

The operation of a polarizer or retarder can be described using Jones matrices. An easy way of presenting monochromatic polarized light is by means of an electric vector written in column representation, J, developed by R. Clark Jones in 1941 and called the Jones vector. In a similar manner an optical system which transforms a $\mathbf{J}_{1}$ state into a $\mathbf{J}_{\mathbf{2}}$ state can be written in terms of a $2 \times 2$ matrix, known as the Jones matrix, $\mathbf{T}$, and then it is possible to write $\mathbf{J}_{2}=\mathbf{T} \mathbf{J}_{1}$. Working with matrices has the advantage that the action of optical systems can be represented by a matrix given by the product of the Jones matrices of the different elements which make up the system. This method has great potential for students since a large number of optical systems can easily be analyzed by performing matrix multiplication.

This is a general method for presenting polarized light and birefringence using an experimental device composed of a birefringent plate placed between a polarizer and an analyzer. After passing through the polarizer the light is linearly polarized in the direction given by the transmission axes of the polarizer. The electric field can be decomposed into two orthogonal axes $x$ and $y$, and after light passes through the retarder (birefringent plate) a phase shift $\Gamma$ is introduced between the $x$ component, $E_{x}$, and the $y$ component, $E_{y}$, of the electric field. The light then passes through the analyzer with the transmission axes forming an angle $\theta$ with the transmission axes of the polarizer. The intensity as a function of the angle $\theta$ can be measured and the value of the retardation angle $\Gamma$ can be obtained by making a theoretical fit to the experimental data.

In this paper we analyze, theoretically and experimentally, the birefringence of a retarder plate. Following Blanco et al [3] we use strips of cellophane tape as the retarder plate and verify that this material behaves like a uniaxial crystal. The use of cellotape in polarization demonstrations has already reported in several educational papers. The use of cellotape to study birefringence was proposed by Feymnan [3, 5] and an interesting photograph of a strip of Scotch tape placed between two crossed polarizers can be found in the third edition of Hecht's book on Optics [6]. Babic and Cepic [7] use cellophane to generate colours and Velasques et al [8] employ the Jones representation to analyze 
cellophane tape retarders and the experimental measurements are carried out using a broadband light source and a spectrometer. In our experiments the cellotape is placed between two polarizers and illuminated with monochromatic light at different wavelengths. Measuring the intensity through the system it is possible to obtain information about the characteristics of the cellotape as a retarder plate. The experiment described, although very simple, has great potential since different concepts relating to polarization of light can easily be studied. In this paper we propose another simple experiment which is based on the one described, although some changes have been made. This enriches the laboratory experience and permits the students to practice using matrix representation. I think this work can be interesting for experimental demonstration to undergraduate students in courses on Optics.

\section{Theoretical analysis}

Consider an experimental device such as that represented in Figure 1. The device is composed of a birefringent crystal plate placed between a polarizer and an analyzer. After passing through the polarizer, the light is linearly polarized in the direction given by the transmission axes of the polarizer. The electric field can be decomposed into two orthogonal axes $x$ and $y$, and after light passes through the retarder (birefringent plate) a phase shift $\Gamma$ is introduced between the $x$ component, $E_{x}$, and the $y$ component, $E_{y}$, of the electric field. The light then passes through the analyzer and is again linearly polarized. In this section the optical system (polarizer-retarder-analyzer) is characterized by obtaining its Jones matrix. As can be seen from the figure, the transmission axis of the polarizer coincides with axis $x$, the angle introduced by the retarder will be $\Gamma$, and its optical axis forms an angle $\alpha$ with the $x$ axis. Finally, the transmission axis of the analyzer forms an angle $\theta$ with the $x$ axis. The Jones matrix of the system can be calculated as follows

$$
\mathbf{H}=\mathbf{T}_{3} \mathbf{T}_{2} \mathbf{T}_{1}
$$


where $\mathbf{T}_{3}$ is the matrix corresponding to the analyzer, $\mathbf{T}_{2}$ the matrix of the retarder and $\mathbf{T}_{1}$ that of the linear polarizer. For a linear polarizer with the transmission axis along the $x$ axis, the Jones matrix is given as follows

$$
\mathbf{T}=\left[\begin{array}{ll}
1 & 0 \\
0 & 0
\end{array}\right]
$$

On the other hand, the transmission axis of the analyzer forms an angle $\theta$ with the $x$ axis, whereas the optical axis of the retarder is also allowed to form an angle $\alpha$ with the $x$ axis. If a Jones matrix, $\mathbf{T}^{\prime}$, is obtained in a system $x^{\prime} y^{\prime}$ which is rotated through an angle $\beta$ with respect to the axis $x y$, the matrix $\mathbf{T}$ in system $x y$ is given by

$$
\mathbf{T}=\mathbf{R}(\beta) \mathbf{T}^{\prime} \mathbf{R}(-\beta)
$$

where $\mathbf{R}(\beta)$ is the 2 x 2 rotation matrix given by

$$
\mathbf{R}(\beta)=\left[\begin{array}{cc}
\cos \beta & \sin \beta \\
-\sin \beta & \cos \beta
\end{array}\right]
$$

Therefore, the Jones matrix $\mathbf{T}_{\mathbf{3}}$ corresponding to the analyzer, which is a linear polarizer with a transmission axis making an angle $\theta$ with the $x$ axis is

$$
\mathbf{T}_{3}=\mathbf{R}(\theta) \mathbf{T}_{3}^{\prime} \mathbf{R}(-\theta)=\left[\begin{array}{cc}
\cos \theta & \sin \theta \\
-\sin \theta & \cos \theta
\end{array}\right]\left[\begin{array}{ll}
1 & 0 \\
0 & 0
\end{array}\right]\left[\begin{array}{cc}
\cos \theta & -\sin \theta \\
\sin \theta & \cos \theta
\end{array}\right]=\left[\begin{array}{cc}
\cos ^{2} \theta & \sin \theta \cos \theta \\
\sin \theta \cos \theta & \sin ^{2} \theta
\end{array}\right]
$$

The matrix $\mathbf{T}_{2}^{\prime}$ corresponding to the wave retarder in the system $x^{\prime} y^{\prime}$ (because we measure intensities, we can assume the fast axis is along the $x^{\prime}$ direction) is

$$
\mathbf{T}_{2}^{\prime}=\left[\begin{array}{cc}
1 & 0 \\
0 & \exp (-i \Gamma)
\end{array}\right]
$$


and then, in the $x y$ system it is possible to write

$$
\mathbf{T}_{2}=\mathbf{R}(\alpha) \mathbf{T}_{2}^{\prime} \mathbf{R}(-\alpha)=\left[\begin{array}{cc}
\cos \alpha & \sin \alpha \\
-\sin \alpha & \cos \alpha
\end{array}\right]\left[\begin{array}{cc}
1 & 0 \\
0 & \exp (-i \Gamma)
\end{array}\right]\left[\begin{array}{cc}
\cos \alpha & -\sin \alpha \\
\sin \alpha & \cos \alpha
\end{array}\right]
$$

The final matrix of the system (polarizer-retarder-analyzer) can be calculated as follows

$\mathbf{H}(\alpha, \theta)=\left[\begin{array}{cc}\cos ^{2} \theta & \sin \theta \cos \theta \\ \sin \theta \cos \theta & \sin ^{2} \theta\end{array}\right]\left[\begin{array}{cc}\cos \alpha & \sin \alpha \\ -\sin \alpha & \cos \alpha\end{array}\right]\left[\begin{array}{cc}1 & 0 \\ 0 & \exp (-i \Gamma)\end{array}\right]\left[\begin{array}{cc}\cos \alpha & -\sin \alpha \\ \sin \alpha & \cos \alpha\end{array}\right]\left[\begin{array}{ll}1 & 0 \\ 0 & 0\end{array}\right](9)$

The matrix $\mathbf{H}$ can be simplified and we obtain

$$
\mathbf{H}(\alpha, \theta)=[\cos \alpha \cos (\alpha+\theta)+\exp (-j \Gamma) \sin \alpha \sin (\alpha+\theta)]\left[\begin{array}{ll}
\cos \theta & 0 \\
\sin \theta & 0
\end{array}\right]
$$

Once the matrix which characterizes the optical system is obtained, the output Jones vector $\mathbf{J}_{2}$ is calculated by multiplying $\mathbf{H}$ by the Jones vector of the incident light

$$
\mathbf{J}_{1}=\left[\begin{array}{l}
A_{1 x} \\
A_{1 y}
\end{array}\right]
$$

The output intensity can be obtained using the equation

$$
I(\alpha, \theta)=\frac{1}{2} \varepsilon_{0} c\left(\left|A_{2 x}\right|^{2}+\left|A_{2 y}\right|^{2}\right)
$$

where $\varepsilon_{0}$ is the permittivity of free space, $c$ is the velocity of light and 


$$
\mathbf{J}_{2}=\left[\begin{array}{c}
A_{2 x} \\
A_{2 y}
\end{array}\right]
$$

where $\mathbf{J}_{2}=\mathbf{H} \mathbf{J}_{1}$. After some manipulations, and taking into account that the polarization of the light incident on the first polarizer is given by $\mathbf{J}_{1}=\left(\begin{array}{l}1 \\ 0\end{array}\right)$, the calculated intensity can be written as follows

$$
I(\alpha, \theta)=\frac{1}{2} I_{0}\left[1+\left(\cos ^{2} 2 \alpha+\cos \Gamma \sin ^{2} 2 \alpha\right) \cos 2 \theta+\sin ^{2}\left(\frac{\Gamma}{2}\right) \sin 4 \alpha \sin 2 \theta\right]
$$

where $I_{0}$ denotes the maximum intensity transmitted by the whole system and for the experimental measurements $I_{0}$ will also take into account the losses that occur in the different elements of the system (polarizer, cellotape and retarder). The intensity at the output of the optical system is, then, a function of the angle $\theta$ between the transmission axis of the analyzer and the plane of polarization of the incident light; of $\alpha$, which is the angle formed by the optical axis of the retarder and the $x$ axis; and of $\Gamma$, the retardation angle introduced by the retarder. If we define $x=\cos 2 \theta, y=I$ and $y_{0}=I_{0}$, equation (14) can be written as follows

$$
x^{2}+4\left(y-\frac{y_{0}}{2}\right)^{2}-4\left(\cos ^{2} 2 \alpha+\cos \Gamma \sin ^{2} 2 \alpha\right) x\left(y-\frac{y_{0}}{2}\right)=\sin ^{4}\left(\frac{\Gamma}{2}\right) \sin ^{2} 4 \alpha
$$

which, in general, corresponds to an ellipse in the $x y$ plane whose center is the point with coordinates $\left(0, I_{0} / 2\right)$. Then, if $I$ is plotted as a function of $\cos 2 \theta$, an ellipse is obtained. An interesting case can to study is when $\alpha=45^{\circ}$. In this case, from equation (14) it is easy to verify that there is a linear relation between the intensity, $I$, and $\cos 2 \theta$

$$
I=\frac{1}{2} I_{0}(1+\cos \Gamma \cos 2 \theta)
$$


and the ellipse in equation (15) is transformed into a straight line

$$
y=m x+n
$$

where

$$
m=\frac{1}{2} I_{0} \cos \Gamma
$$

and

$$
n=\frac{1}{2} I_{0}
$$

From equations (18) and (19) it is possible to obtain the value $\cos \Gamma$ from intensity measurements, as follows

$$
\cos \Gamma=\frac{m}{n}
$$

This allows us to obtain the value of the phase $\Gamma$ knowing the values of $m$ and $n$, which can be calculated from a least square fit to the experimental data of the intensity as a function of $\cos 2 \theta$, when $\alpha=45^{\circ}$.

\section{Experimental and results}

As mentioned above, different contributions of great interest may be found in the literature describing simple experiments which enable the students to understand how a retarder works and to determine the retardation introduced by a uniaxial crystal [3]. As in these references, we used cellotape as the birefringement material and an optic system consisting of a linear polarizer and analyzer, both mounted on rotation graduated stages, with the cellotape placed between them and also mounted on a graduated rotation stage. The different elements making up the system are located along the $z$ axis which coincides with the direction of propagation of light. After light passes through the system we measured the irradiance by means of a detector. Figure 2 shows the graduated rotation stage where the cellotape was placed. We used four $\mathrm{cw}$ lasers of wavelengths $632.8 \mathrm{~nm}$ 
(red HeNe laser), $594 \mathrm{~nm}$ (yellow HeNe laser), $543.5 \mathrm{~nm}$ (green HeNe laser) and $442 \mathrm{~nm}$ (blue HeCd laser). Firstly, we used equation (16) to adjust the value of $\alpha=45^{\circ}$. To do this we selected $\alpha=45^{\circ}$ on the rotation stage where the strip of cellotape is placed. Then we measured the intensity as a function of $\cos \theta$ and slightly modified the value of $\alpha$ with the help of the micrometric screw to obtain a linear relation between $I$ and $\cos 2 \theta$, as shown in equation (17). This reference is considered the direction of the optic axis of the cellotape for $\alpha=45^{\circ}$ and it allowed us to easily select the other values of the angle $\alpha$. We can also find the neutral axes of the retarder in a much simpler way by placing it between crossed polarizers and seeking the null transmission.

In our first experiments, for each wavelength, we chose a value of $\alpha$ of $45^{\circ}$ and measured the intensity $I$ through the whole system rotating the angle $\theta$ of the analyzer between $0^{\circ}$ and $180^{\circ}$, and taking measurement every $5^{\circ}$ (37 measurements). Then we plotted the values of $I$ as a function of $\cos 2 \theta$, with $\alpha=45^{\circ}$, and obtained the values of $m$ and $n$, which can be calculated from a least square fit to the experimental data of the intensity as a function of $\cos 2 \theta$. Table I and Figure 3 show the results obtained. From this figure it is clear that for $\alpha=45^{\circ}$ there is a linear relation between $I$ and $\cos 2 \theta$.

However, the cosine alone is not sufficient to define the angle, and so the value of the phase difference $\Gamma$ can not be obtained from this value alone [3]. To obtain the true value of the phase difference we proceeded as follows. If $\Gamma$ is the true value of the phase difference, its value will be that given in equation (1), where we can only ensure that $\Delta n$ $=\left|n_{e}-n_{o}\right|, \quad n_{e}$ and $n_{o}$ being the refractive indexes for the extraordinary and ordinary waves, respectively. Even though $\Delta n$ itself is wavelength dependent, we disregarded any variation of $\Delta n$ with wavelength because it is very small, and then it may be assumed that the retardation angle $\Gamma$ is inversely proportional to the wavelength $\lambda_{0}$. We verified that this supposition is approximately correct for the interval of wavelengths considered. Knowing the value of $\cos \Gamma$ from the least square fit, we obtain the smallest possible value $\Gamma_{1}$ of the phase difference $\Gamma$ is given by

$$
\Gamma_{1}=\cos ^{-1}(\cos \Gamma) \quad \text { with } \quad 0 \leq \Gamma_{1}<\pi
$$


Then, the relation between the true and the smallest possible values for the phase difference can be written as follows

$$
\Gamma=2 \pi N \pm \Gamma_{1} \quad \text { with } \quad 0 \leq \Gamma_{1}<\pi
$$

where $N$ is a non-negative integer. In order to resolve the ambiguities in the values of the phase shifts we consider equation (20), which allows us to write (disregarding any variation of $\Delta n$ with wavelength)

$$
\Gamma_{\text {blue }}>\Gamma_{\text {green }}>\Gamma_{\text {yellow }}>\Gamma_{\text {red }}
$$

and

$$
\frac{\Gamma_{j}}{\Gamma_{\text {blue }}}=\frac{\lambda_{\text {blue }}}{\lambda_{j}}
$$

From equations (20)-(24) it may be easily verified that a value of $N=1$ and the minus sign must be selected in equation (22). In Table 2 we present the different steps followed to obtain the true value of the retardation angle.

In a subsequent experiment, and for a wavelength of $633 \mathrm{~nm}$, we measured the intensity as a function of the angle $\theta$ but for different values of $\alpha\left(0^{\circ}, 10^{\circ}, 20^{\circ}, 30^{\circ}, 40^{\circ}\right.$ and $45^{\circ}$ ). In Figure 4 we plotted the measured intensity as a function of $\cos 2 \theta$. In this figure the dots represent the experimental values, whereas the continuous lines correspond to the theoretical values obtained using equation (13) with the values of $I_{0}$ and $\cos \Gamma$ obtained previously. We can see in this figure the different types of ellipses obtained. Figure 5 shows the measured intensity as a function of $\alpha$ for different values of $\theta$, whereas in Figure 6 we plotted the measured intensity as a function of $\theta$ for different values of $\alpha$. All these figures show good agreement between experimental and theoretical values, which verifies that the behaviour of the cellotape layer used is like that of a uniaxial crystal. 
Finally in order to verify our hypothesis that $\Delta n$ does not depend on the wavelength $\lambda_{0}$, we plotted $\Gamma$ as a function of $\lambda_{0}^{-1}$ taking into account equation (20). As can be seen in Figure 7 there is practically a linear relation between them. From this figure we can do a least square fit to the experimental data and obtain an experimental value for $\Delta n d$ for a single layer of cellotape, which is $326 \pm 2 \mathrm{~nm}$. Finally, we obtained the thickness $d$ of the cellotape measuring the thickness of 16 layers and found that $16 d=$ $0.68 \pm 0.02 \mathrm{~mm}$ and then $d=42.5 \pm 1.3 \mu \mathrm{m}$, which allows us to conclude that the birefringence of the cellotape analyzed is $\Delta n=0.0077 \pm 0.0003$ for the interval of wavelengths considered. This result is only an approximation, since the difference between refractive indices, $\Delta n$, suffers in general from chromatic dispersion. However, the results in Figure 7 suggest that there is not dispersion and we can provide in fact a single value for $\Delta n$. This can be a surprising result for the reader, but we can explain it considering now that $\Delta n$ depends on the wavelength $\lambda_{0}$. A good approximation to the wavelength dependence of $\Delta n$ is the Cauchy-type equation [7]

$$
\Delta n=a+\frac{b}{\lambda_{0}^{2}}
$$

where $a$ and $b$ are constant parameters. Then we can write Eq. (1) as follows

$$
\Gamma=\frac{2 \pi}{\lambda_{0}} \Delta n d=\frac{A}{\lambda_{0}}+\frac{B}{\lambda_{0}^{3}}
$$

Our fit of the experimental data to Eq. (26) gives $A=2036 \mathrm{~nm} / \mathrm{rad}$ and $B=2.8326 \times 10^{6}$ $\mathrm{nm}^{3} / \mathrm{rad}$ and we obtain $a=0.007624$ and $b=10.61 \mathrm{~nm}^{2}$, which allows us to write Eq. (25) as follows

$$
\Delta n=0.007624+\frac{10.61}{\lambda_{0}^{2}}
$$


which takes values from 0.007678 to 0.007650 for wavelengths between $442 \mathrm{~nm}$ and $632.8 \mathrm{~nm}$. These values can be approximated writing $\Delta n=0.0077 \pm 0.0003$, which is the value previously obtained. Figure 8 shows the experimental values for the birefringence of a single cellotape layer and the theoretical fits obtained considering that $\Delta n$ does nor depend on the wavelength and that the wavelength dependence of $\Delta n$ is the Cauchy-type equation.

\section{Conclusions}

A simple system consisting of a strip of cellotape placed between two polarizers was used to analyze the behaviour of this cellotape layer as a birefringent retarder. The system was firstly theoretically analyzed using Jones matrices, which allows us to easily obtain an expression for the intensity transmitted by the whole system as a function of the angles between the optic axis of the retarder and the transmission axis of the analyzer, respectively, and the transmission axis of the polarizer. The use of monochromatic light of different wavelengths allowed us to obtain the true value of the retardation angle by measuring the transmitted intensity for an angle of $45^{\circ}$ between the optic axis of the retarder and the transmission axis of the polarizer. Finally, the value of $\Delta n d$ for a single layer of cellotape is also obtained.

\section{Acknowledgements}

This work was supported by the 'Ministerio Ciencia e Innovación' of Spain under projects FIS2008-05856-C02-01 and FIS2008-05856-C02-02, by the 'Vicerrectorado de Tecnología e Innovación Educativa' of the University of Alicante, Spain (GITE-09006UA). 
A. Beléndez, E. Fernández, J. Francés and C. Neipp, "Birefringence of cellotape: Jones representation and experimental analysis", European Journal of Physics, Vol. 31, № 3, pp. 551-561 (2010). doi:10.1088/0143-0807/31/3/012

ISSN 0143-0807 (Print) ISSN 1361-6404 (Online)

\section{References}

[1] Matveev A N 1988, Optics, Mir Publishers, Moscow. Chap. 8.

[2] Guenther R. 1990, Modern Optics, John Wiley \& Sons. New York. Chap. 13.

[3] Blanco M A, Yuste M and Carreras C 1997, Undergraduate experiment designed to show the proportionality between the phase difference and the thickness of a uniaxial crystal Am. J. Phys. 65, 784-7.

[4] Saleh B E A and Teich M C 1991, Fundamentals of Photonics, John Wiley \& Sons, New York. Chap. 6.

[5] Feymnan R, Leighton R B and Sands M L 1989, The Feynman Lectures on Physics, Commemorative Issue, California Institute of Technology, Reading, MA. Vol. I. Chap. 33.

[6] Hecht E 1998, Optics, Addison Wesley Longmann, Inc. Chap. 8.

[7] Babic V, Cepic M 2009 Complementary colours for a physicist Eur. J. Phys. 30, 793803

[8] Velasquez P, Sánchez-lópez M M, Moreno I, Puerto D, Mateos F 2005, Interference birefringent filters fabricated with low cost commercial polymers Am. J. Phys. 73, 357-61 


\section{FIGURE CAPTIONS}

Figure 1.- Experimental set up.

Figure 2.- Photograph of the cellotape on the rotation stage.

Figure 3.- Measured values of the intensity (dots) as a function of $\cos 2 \theta$ for $\alpha=45^{\circ}$ and linear fit (continuous lines) for the four wavelengths considered.

Figure 4.- Measured intensity as a function of $\cos 2 \theta$ for a wavelength of $632.8 \mathrm{~nm}$ : Experimental measurements (dots) and theoretical values (continuous line) obtained considering $I_{0}=15.6 \pm 0.4$ (a.u.) and $\cos \Gamma=-0.995 \pm 0.005$ in equation (13).

Figure 5.- Measured intensity as a function of $\alpha$ for different values of $\theta$ and a wavelength of $632.8 \mathrm{~nm}$.

Figure 6.- Measured intensity as a function of $\theta$ for different values of $\alpha$ and a wavelength of $632.8 \mathrm{~nm}$.

Figure 7.- Retardation angle $\Gamma$ as a function of $1 / \lambda_{0}$ and linear fit.

Figure 8.- Birefringence versus de wavelength of a single cellotape layer. Experimental (dots), fitting considering that $\Delta n$ is not a function of the wavelength (dashed line) and fitting considering that the wavelength dependence of $\Delta n$ is the Cauchy-type equation (continuous line). 
A. Beléndez, E. Fernández, J. Francés and C. Neipp, "Birefringence of cellotape: Jones representation and experimental analysis", European Journal of Physics, Vol. 31, № 3, pp. 551-561 (2010). doi:10.1088/0143-0807/31/3/012

ISSN 0143-0807 (Print) ISSN 1361-6404 (Online)

\section{TABLES}

Table 1.- Values of $m, n$ and $\cos \Gamma$, which can be calculated from a least square fit to the experimental data of the intensity as a function of $\cos 2 \theta$, for $\alpha=45^{\circ}$.

Table 2.- Determination of the true value of the retardation angle. 
A. Beléndez, E. Fernández, J. Francés and C. Neipp, "Birefringence of cellotape: Jones representation and experimental analysis", European Journal of Physics, Vol. 31, № 3, pp. 551-561 (2010). doi:10.1088/0143-0807/31/3/012 ISSN 0143-0807 (Print) ISSN 1361-6404 (Online)

\section{FIGURE 1}



FIGURE 2

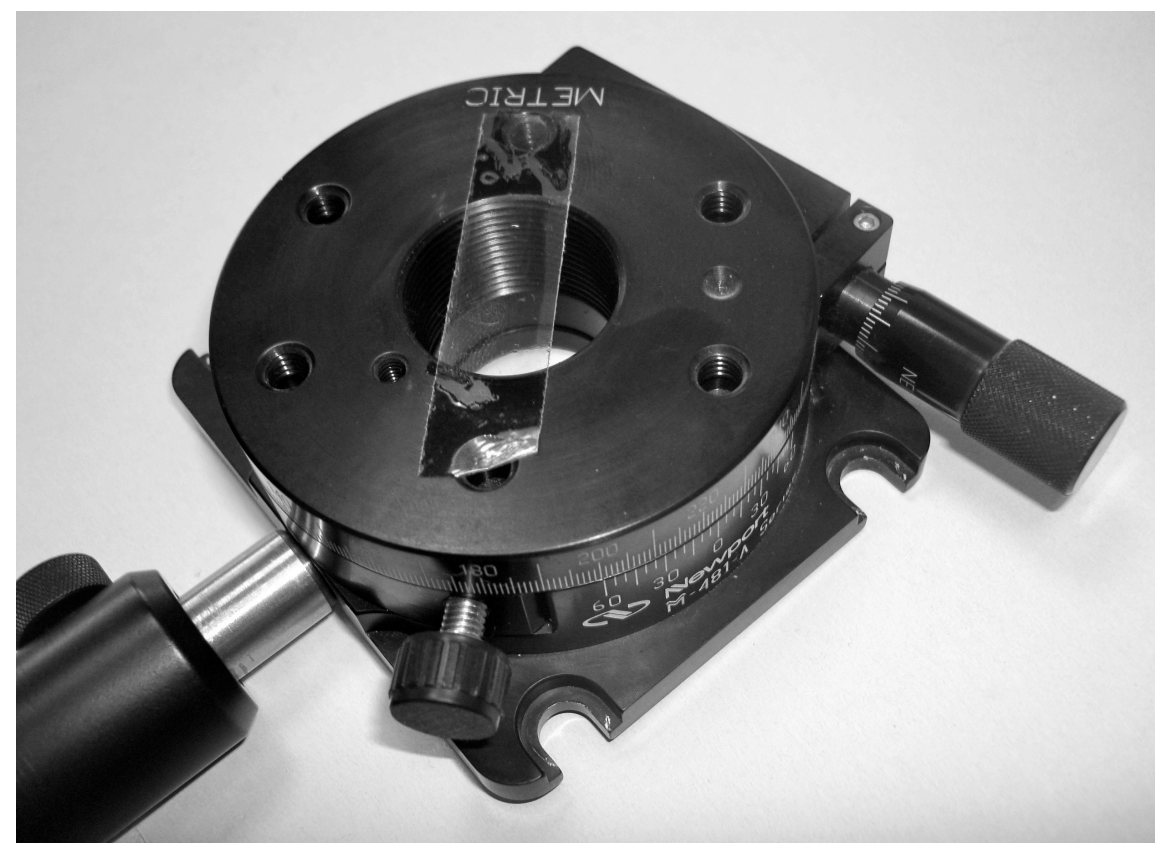


A. Beléndez, E. Fernández, J. Francés and C. Neipp, "Birefringence of cellotape: Jones representation and experimental analysis", European Journal of Physics, Vol. 31, № 3, pp. 551-561 (2010). doi:10.1088/0143-0807/31/3/012

ISSN 0143-0807 (Print) ISSN 1361-6404 (Online)

FIGURE 3
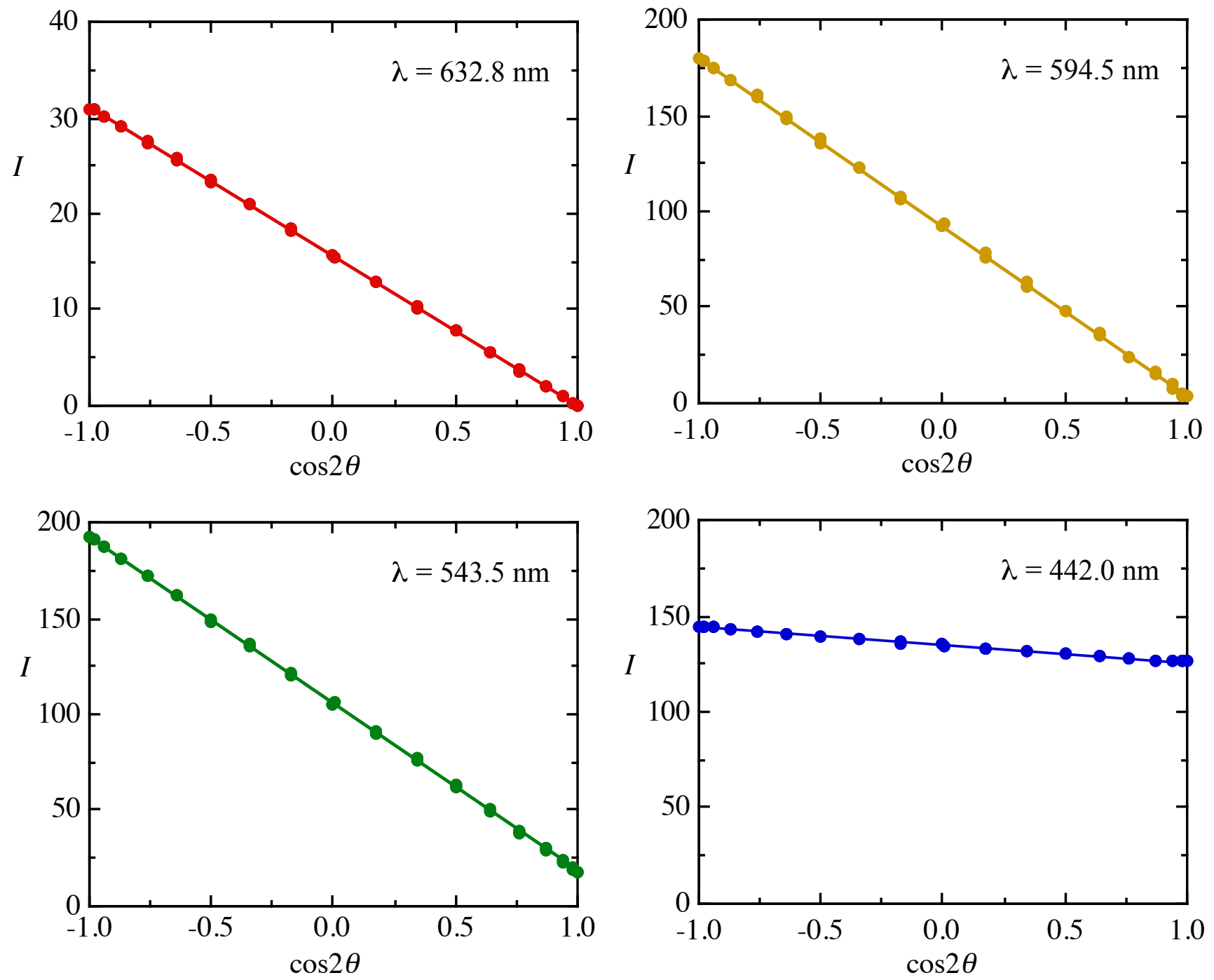
A. Beléndez, E. Fernández, J. Francés and C. Neipp, "Birefringence of cellotape: Jones representation and experimental analysis", European Journal of Physics, Vol. 31, № 3, pp. 551-561 (2010). doi:10.1088/0143-0807/31/3/012

ISSN 0143-0807 (Print) ISSN 1361-6404 (Online)

\section{FIGURE 4}
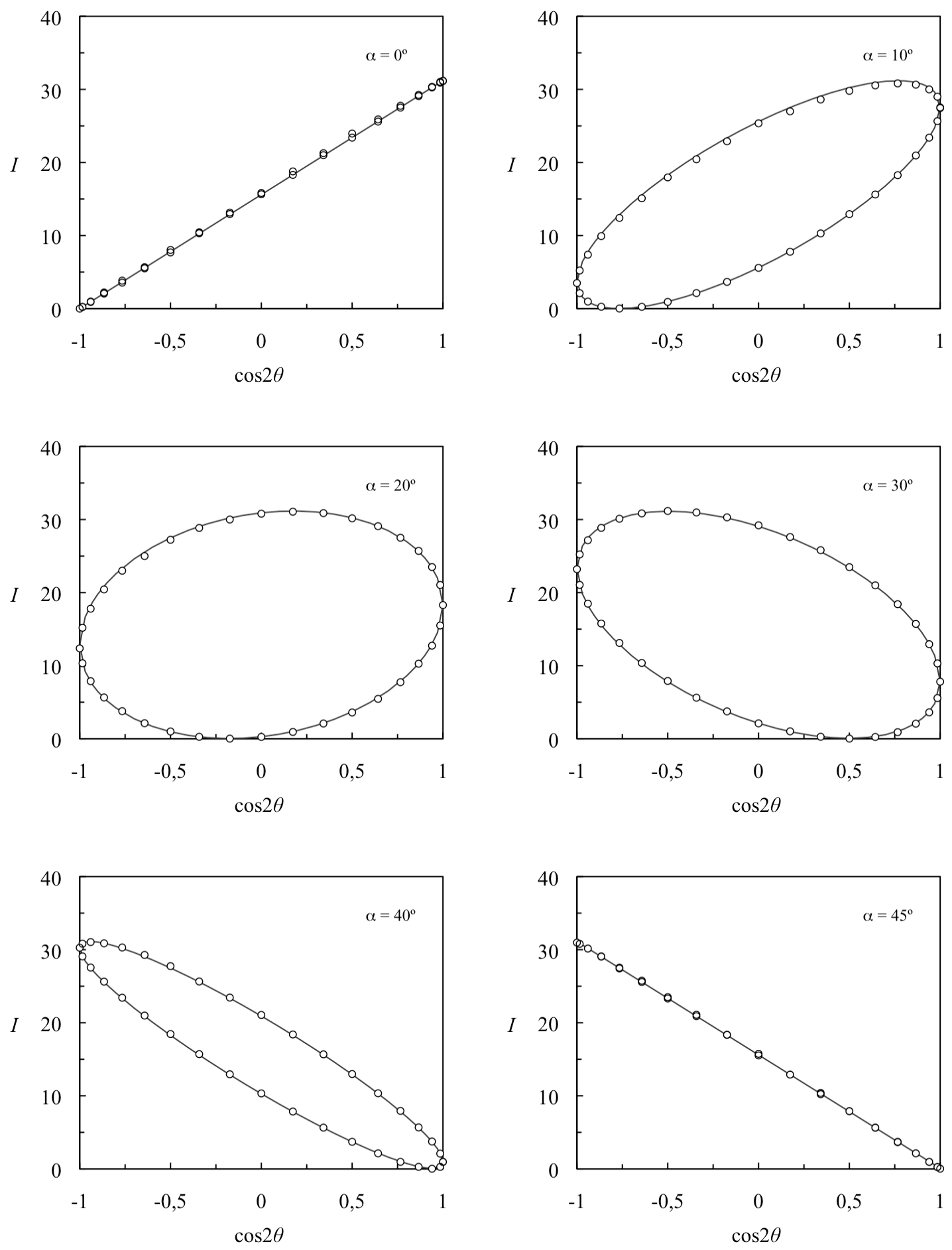
A. Beléndez, E. Fernández, J. Francés and C. Neipp, "Birefringence of cellotape: Jones representation and experimental analysis", European Journal of Physics, Vol. 31, № 3, pp. 551-561 (2010). doi:10.1088/0143-0807/31/3/012

ISSN 0143-0807 (Print) ISSN 1361-6404 (Online)

\section{FIGURE 5}

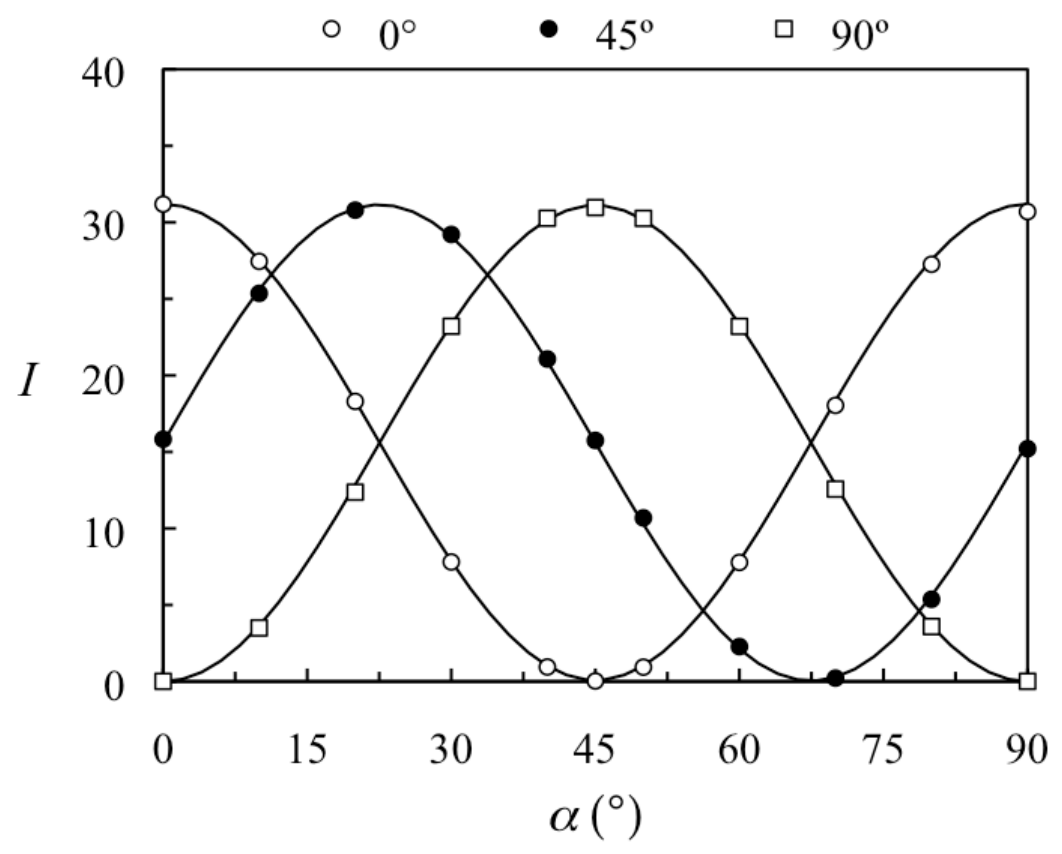

FIGURE 6

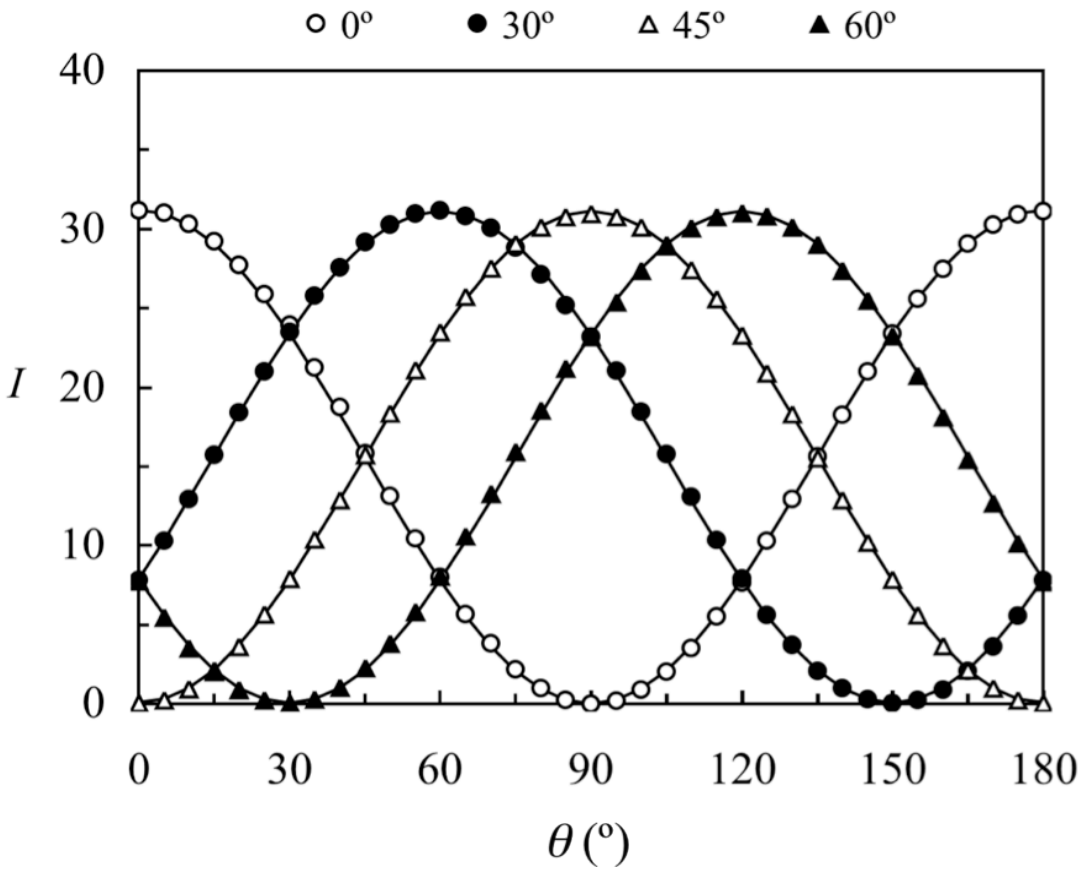


A. Beléndez, E. Fernández, J. Francés and C. Neipp, "Birefringence of cellotape: Jones representation and experimental analysis", European Journal of Physics, Vol. 31, № 3, pp. 551-561 (2010). doi:10.1088/0143-0807/31/3/012 ISSN 0143-0807 (Print) ISSN 1361-6404 (Online)

\section{FIGURE 7}

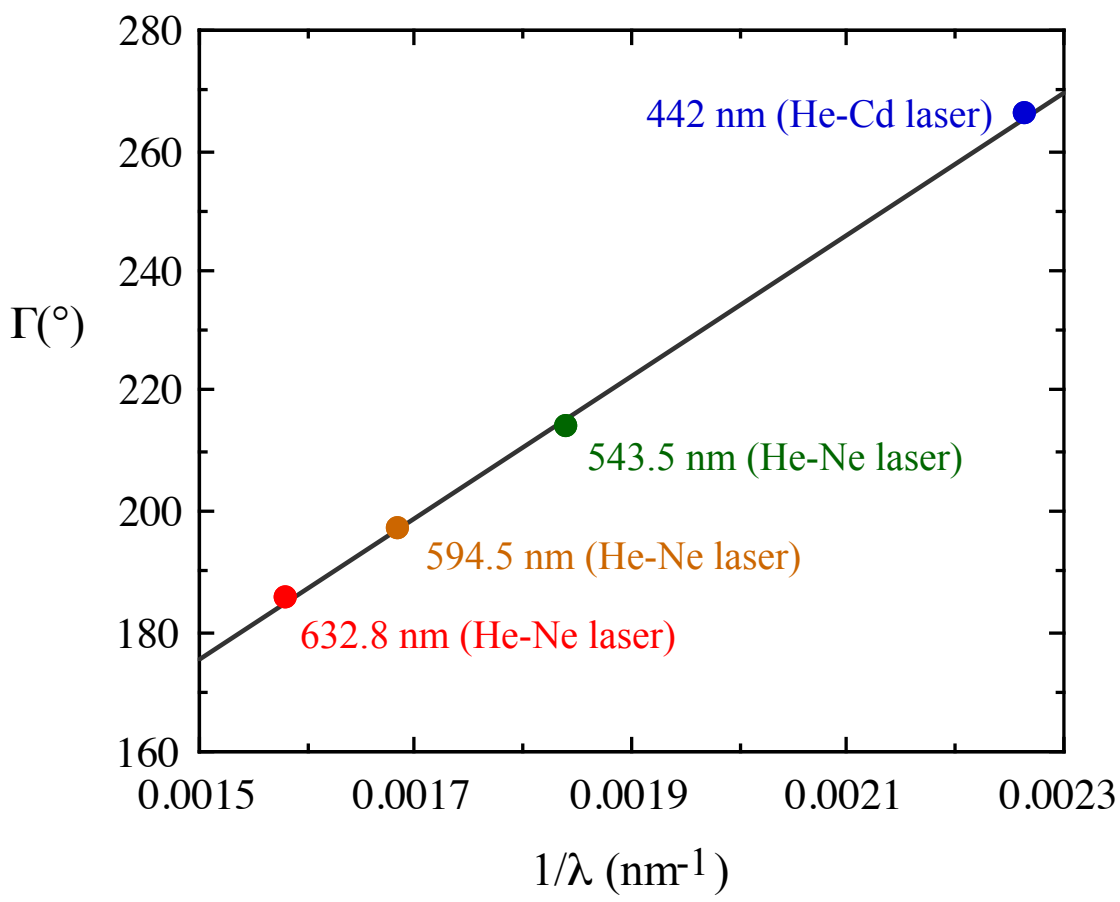

FIGURE 8 
A. Beléndez, E. Fernández, J. Francés and C. Neipp, "Birefringence of cellotape: Jones representation and experimental analysis", European Journal of Physics, Vol. 31, № 3, pp. 551-561 (2010). doi:10.1088/0143-0807/31/3/012

ISSN 0143-0807 (Print) ISSN 1361-6404 (Online)

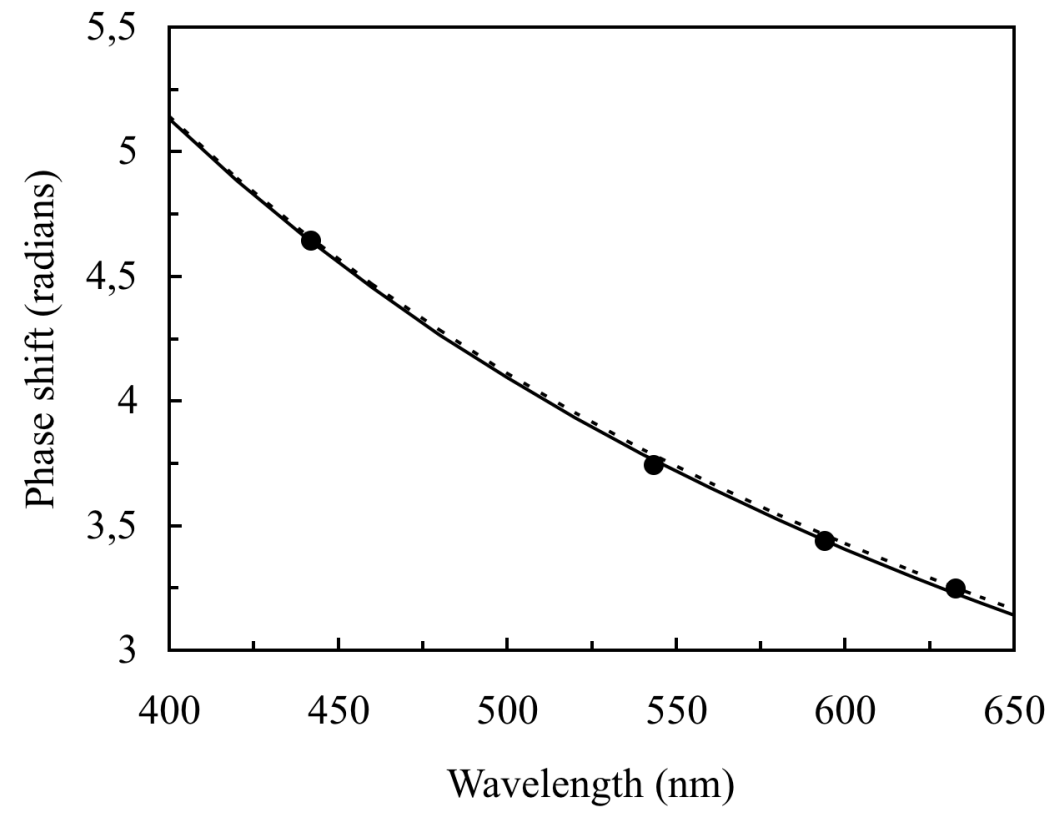

TABLE 1

\begin{tabular}{llll}
\hline$\lambda_{0}(\mathrm{~nm})$ & $m$ & $n$ & $\cos \Gamma$ \\
\hline 442.0 & $-9.3 \pm 0.4$ & $135.1 \pm 1.3$ & $-0.0686 \pm 0.0024$ \\
543.5 & $-87 \pm 3$ & $106 \pm 3$ & $-0.825 \pm 0.010$ \\
594.0 & $-88 \pm 3$ & $92 \pm 3$ & $-0.958 \pm 0.009$ \\
632.8 & $-15.5 \pm 0.4$ & $15.6 \pm 0.4$ & $-0.995 \pm 0.005$ \\
\hline
\end{tabular}

TABLE 2 
A. Beléndez, E. Fernández, J. Francés and C. Neipp, "Birefringence of cellotape: Jones representation and experimental analysis", European Journal of Physics, Vol. 31, № 3, pp. 551-561 (2010). doi:10.1088/0143-0807/31/3/012

ISSN 0143-0807 (Print) ISSN 1361-6404 (Online)

\begin{tabular}{ccccc}
\hline$\lambda_{0}(\mathrm{~nm})$ & $\Gamma_{1}$ & $\left(\Gamma_{j} / \Gamma_{\text {blue }}\right)_{\text {theoretical }}=\lambda_{j} / \lambda_{\text {blue }}$ & $\left(\Gamma_{j}\right)_{\text {experimental }}$ & $\left(\Gamma_{j} / \Gamma_{\text {blue }}\right)_{\text {experimental }}$ \\
\hline 442.0 & $93.9^{\mathrm{o}} \pm 0.2^{\mathrm{o}}$ & 1 & $266.1^{\mathrm{o}} \pm 0.2^{\mathrm{o}}$ & 1 \\
543.5 & $145.6^{\mathrm{o}} \pm 0.4^{\mathrm{o}}$ & 0.8132 & $214.4^{\mathrm{o}} \pm 0.4^{\mathrm{o}}$ & $0.806 \pm 0.002$ \\
594.0 & $163^{\mathrm{o}} \pm 2^{\mathrm{o}}$ & 0.7441 & $197^{\mathrm{o}} \pm 2^{\mathrm{o}}$ & $0.740 \pm 0.008$ \\
632.8 & $174^{\mathrm{o}} \pm 3^{\mathrm{o}}$ & 0.6985 & $186^{\mathrm{o}} \pm 3^{\mathrm{o}}$ & $0.699 \pm 0.012$ \\
\hline
\end{tabular}

